IIIIIIIIIIIIIIIII

Review

IIIIIIIIIIIIIIIII

\title{
Strigolactones-a novel class of phytohormones as anti-cancer agents
}

\author{
Mohammed Nihal Hasan, ${ }^{1}$ Syed S. I. Razvi, ${ }^{1}$ Abudukadeer Kuerban, ${ }^{1}$ \\ Khadijah Saeed Balamash, ${ }^{1}$ Widad M. Al-Bishri ${ }^{1}$ Khalid Omar Abulnaja, $, 13,4$ \\ Hani Choudhry, ${ }^{1,2,4}$ Jehan A. Khan, ${ }^{5}$ Said Salama Moselhy, ${ }^{1,3,4,7, *}$ Zamzami Ma, ${ }^{1,2}$ \\ Taha A. Kumosani ${ }^{1,3,6}$ Abdulrahman L. Al-Malki, ${ }^{1,3,4}$ \\ Mahmoud AlHosin ${ }^{1,2}$ and Tadao AsAmi ${ }^{1,2,8}$ \\ ${ }^{1}$ Department of Biochemistry, Faculty of Science, King Abdulaziz University, Jeddah, Saudi Arabia \\ ${ }^{2}$ Department of Biochemistry, Faculty of Science, King Abdulaziz University, \\ Cancer Metabolism and Epigenetic Unit, Faculty of Science, \\ Cancer and Mutagenesis Unit, King Fahd Medical Research Center, Jeddah, Saudi Arabia \\ ${ }^{3}$ Department of Biochemistry, Faculty of Science, King Abdulaziz University, \\ Experimental Biochemistry Unit, King Fahd Medical Research Center, Jeddah, Saudi Arabia \\ ${ }^{4}$ Department of Biochemistry, Faculty of Science, King Abdulaziz University, \\ Bioactive Natural Products Research Group, Jeddah, Saudi Arabia \\ ${ }^{5}$ Department of Biochemistry, Faculty of Science, King Abdulaziz University, \\ Biological Sciences Department (Genomic division), Faculty of Science, Jeddah, Saudi Arabia \\ ${ }^{6}$ Department of Biochemistry, Faculty of Science, King Abdulaziz University, \\ Production of Bioproducts for Industrial Applications Research Group, Jeddah, Saudi Arabia \\ ${ }^{7}$ Department of Biochemistry, Faculty of Science, Ain Shams University, Cairo, Egypt \\ ${ }^{8}$ Graduate School of Agricultural and Life Sciences, The University of Tokyo, Bunkyo, Tokyo 113-8657, Japan
}

(Received December 6, 2017; Accepted February 2, 2018)

\begin{abstract}
Chemotherapy shows some promising results in the inhibition of cancer, but resistance to chemotherapy and its severe side effects may occur in due course, resulting in only restricted and narrow benefits. Therefore, there is a pressing need to find alternative chemotherapeutic drugs for combating cancers. Plants have been used since ages in medicine, and by the dawn of 19th century, various potent and promising anti-cancer products have been derived from plants. Strigolactones (SLs) are a novel class of phytohormones involved in regulating the branching of shoots. Recently, many novel synthesized SL analogues have been found to be effective against solid and non-solid tumours. These hormones have been reported to have a unique mechanism of inhibiting cancer cells by lowering their viability and promoting apoptosis and cell death at micromolar concentrations. Therefore, synthetic SL analogues could be future potent anti-cancer drug candidates. Further research is needed to identify and deduce the significance of these synthetic SL analogues. (C) Pesticide Science Society of Japan

Keywords: cancer, phytohormones, synthetic SL analogues, GR24, MEB55, ST362, anti-cancer, apoptosis.
\end{abstract}

\section{Intruduction}

Cancer is considered one of the most common causes of deaths worldwide. According to the World Health Organization (WHO) approximately 14 million new cases were reported in 2012 and 8.8 million deaths in 2015. New cases are expected to increase by around $70 \%$ in the next two decades. ${ }^{1)}$ Among cancer-related deaths, metastatic disease is known to be the leading cause of mortality. Traditional chemotherapy for advanced stages of cancer has only been found satisfactory in specific types,

\footnotetext{
* To whom correspondence should be addressed.

E-mail: moselhy6@hotmail.com

Published online August 7, 2018

(C) Pesticide Science Society of Japan
}

such as childhood leukemia and testicular carcinoma. ${ }^{2)}$ In spite of certain initial responses that seem to be favorable for most cancers, conditions like local or distant relapses associated with resistance to chemotherapy may occur, resulting in limited benefits. ${ }^{2)}$ Therefore, it could be concluded that resistance to therapy along with its adverse effects and poor tolerance are the primary reasons behind treatment failures in cancer. ${ }^{3)}$

Hence, to treat the metastatic growth of tumors, it is desired to have treatment regimens that include novel therapeutic drugs that are efficient and safe, targeting only the proliferating cancer cells, and that are capable of self-renewal and survival after therapy, leaving healthy cells unaffected. In the past few years, researchers have been trying to design treatment regimens with the lowest possible drug dosages, using suitable combinations to create synergistic responses for targeting cancer cells and mini- 
mizing toxic side effects. ${ }^{2,4,5)}$

\section{The role of plants in cancer therapy}

Plants are known to have been used in ancient cultures as foods, spices and also medicines. In the 19th century, the isolation of active plant compounds reached a new level, which led to the discovery of significant drugs. Influential drugs such as analgesics (painkillers), including morphine and codeine from opium (Papaver somniferum L.), and quinine, which has antimalarial, analgesic, antipyretic (fever-reducing), and anti-inflammatory properties, were isolated from Cinchona calisaya and Cinchona succirubra. Additionally, digitoxin, a cardiac glycoside isolated from Digitalis purpurea and Digitalis lanata was used to treat cardiac illness and cancers. ${ }^{6-8)}$ These natural compounds offer considerable potent biological activity, signifying their role in the development of therapeutics.

Approximately $25-28 \%$ of the modern medicines used today are derived (directly or indirectly) from plants, thus demonstrating the fact that plants possess enormous medicinal potential in traditional medicine. ${ }^{9,10)}$ Intriguingly, there is an established history of compounds derived from plants as effective anti-cancer agents. ${ }^{11)}$

Curcumin (diferuloylmethane), a polyphenol, has been extensively studied for its anti-cancer activities. It is produced in the rhizome of Curcuma longa and has demonstrated its anticancer effect against many types of cancer cells in both in vitro and in vivo assays. ${ }^{12)}$ Numerous studies have confirmed that curcumin suppresses of NF- $\kappa \mathrm{B}$, a protein transcription factor that is vital to multiple cancers. This suppression causes a decline in the expression of genes targeted by NF- $\kappa \mathrm{B}$ (cyclin D1 and COX2) thereby causing apoptosis. ${ }^{13,14)}$ Further studies implied that it also causes apoptosis and autophagy when administered together with oestrogen receptor antagonist, such as Tamoxifen, for the treatment of melanoma. Curcumin causes oxidative damage to mitochondrial DNA in human hepatoma G2 cells resulting in the release of cytochrome $\mathrm{c}$ from mitochondria to cytosol, thus activating autophagy. Additionally, previous studies have also reported its impact on the assembly of microtubules leading to a mitotic arrest in cells. ${ }^{15-17)}$

Paclitaxel (Taxol) is among the most well-known anticancer drugs. This taxane (class of diterpenes) is extracted from the bark of Taxus brevifolia and has revealed its cytotoxicity on cancer cells, ${ }^{18)}$ by binding polymerized microtubules and inhibiting their disassembly. ${ }^{19,20)}$ It also changes the conformation of the microtubules to curtail the binding of microtubule-associated proteins (MAPs) to microtubules. As a consequence, these changes disrupt the functions of microtubules, preventing the formation of mitotic spindles, hence, inhibiting mitosis. ${ }^{19,21)}$

Vinblastine is a vinca alkaloid known as a potent anti-cancer drug. It was initially isolated from Catharanthus roseus and is said to be one of the oldest known microtubule-targeting agents. $^{22)}$ In contrast to microtubule-stabilizing agents (Paclitaxel, which enhances microtubule polymerization and inhibits their disassembly), microtubule-destabilizing agents such as vin- blastine inhibit microtubule polymerization, thereby arresting cells in the mitotic stage. ${ }^{23)}$

\section{The role of phytohormones in cancer therapy}

Recently, phytohormones have also demonstrated the ability to inhibit the proliferation and survival of human cancer cell lines. Cytokinins are phytohormones known to be involved in the regulation of plant cell division. Interestingly, they also inhibit the growth of cancer cells and can, therefore, be classified as anticancer agents. $^{24-26)}$

Brassinosteroids, a group of potent steroid hormones are produced by plants. These are involved in a number of functions including cell division, stem elongation, disease resistance, and senescence; impressively, they are also found to have an inhibitory effect on cancer cell lines by regulating cyclin D1 and cyclin E levels, causing G1 arrest and apoptosis. ${ }^{27)}$

Methyl jasmonate (MeJA) is a stress hormone that belongs to the jasmonate family of phytohormones and is known to play essential role in the developmental process and defence responses of plants. Some studies have reported that MeJA acts against cancer by severely depleting of ATP in cancer cells through mitochondrial perturbation and by removing hexokinase from mitochondria. ${ }^{28)}$

\section{Strigolactones-a novel class of phytohormones as anti-cancer agents}

Strigolactones (SLs), a class of phytohormones that contain lactone groups are produced in many plant species. These are involved in the regulation of shoot branching by the inhibition of growth and the self-renewal of the auxiliary meristem. ${ }^{29,30)}$ It was reported in 1966 that these phytohormones were first identified in Striga sp. and are germination stimulants. ${ }^{31)}$ SLs have been implicated in different signaling pathways in plants in biotic and abiotic stress conditions. Different isoforms of SLs may be produced by a single plant species. ${ }^{32)}$ SLs belong to the terpenoid family and are synthesized via the carotenoid synthesis pathway; their molecular structure is made of an $\mathrm{ABC}$ ring system connecting a butenolide via enol ether bridge. ${ }^{32)}$ The regulation of growth by SLs has been shown to be both positive and negative, as they promote the branching of the symbiotic fungi, arbuscular mycorrhizal fungi, and also cause primordial root formation $^{33,34)}$ while impeding the branching of shoots by controlling the growth of axillary meristem cells. ${ }^{29,30,35)}$

Recently, some SL analogues have been synthesized in different laboratories to assess their antiproliferative activities against cancer cells. These synthesized SL analogues were initially tested on breast cancer cell lines, and their inhibitory effects on the survival and growth of cancer cells were reported. In succeeding studies, the anti-proliferative effect was observed in solid tumors such as lung, prostate, colon, osteosarcoma and melanoma and non-solid cancer cell lines, such as leukaemia. Cell viability was reported to be significantly reduced after treatment with synthetic SL analogues.,36) These synthetic SL analogues were shown to instigate $\mathrm{G} 2 / \mathrm{M}$ arrest leading to apoptosis, al- 
A)

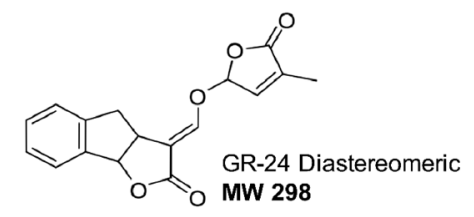

B)

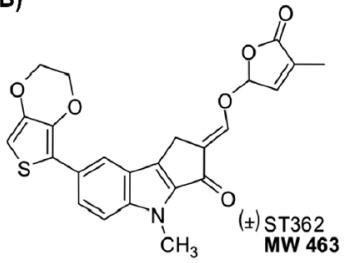

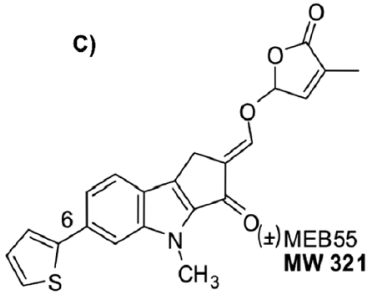

Fig. 1. Molecular structures of synthetic strigolactone analogues GR-24, ST362 and MEB55 with their respective molecular weights. ${ }^{37)}$

though to varying extents with different SL analogues. Synthetic SL analogues were also tested against normal cells (BJ fibroblasts), but only a subtle inhibitory effect was observed with the highest concentration, ${ }^{37)}$ suggesting selective inhibitory activity. The synthetic SL analogue-GR-24 was found to inhibit the growth of different breast cancer cell lines. Although the concentrations used were as low as $2.5-5 \mathrm{ppm}$, there was substantial growth of cancer cells. It was also observed that GR-24 induced G2/M arrest through the upregulation of mitogen-activated protein kinases (MAPKs) p38 and JNK1/2 and promoted apoptosis. p38 and JNK1/2 are stress-activated MAPKs that play a crucial role in stress signalling cascades and are found to be associated with cell cycle arrest ${ }^{38)}$ and apoptosis in some cells. ${ }^{39,40)}$ GR-24 was described as having a dose-dependent effect on tumorigenic breast cancer cells, as a higher number of cells arrested in G2/M phase with a higher concentration of the analogue; however, there was an associated decrease in the cells present in the G1 phase. ${ }^{37)}$ Other synthetic SL analogues, such as ST362 and MEB55 also showed the same anti-proliferative activity against breast cancer cell lines. Strikingly, these synthetic SL analogues also inhibited the viability and growth of mammosphere cultures that were augmented with breast cancer stem-like cells, thereby acting as potent anti-cancer agents. Figure 1 shows the structure of some important SLs, such as GR-24, ST362 and MEB55, with their respective molecular weights.

Cancer stem-like cells aid in the reappearance of components of tumor cells that have been treated with chemotherapy and radiotherapy and are themselves inherently resistant to chemotherapy and radiation therapy, hence it is necessary to inhibit these stem-like cells. ${ }^{37)}$ Furthermore, a study on the treatment of cancer cell lines with synthetic SL analogues (MEB55 and ST362) also found them to be associated with the tested cancer cells being arrested at the G2 phase through the activation of the p38 and JNK1/2 MAPKs causing stress induction and the inhibition of ERK1/2 and PI3K/AKT.,36) The ERK1/2 and PI3K/ AKT pathways regulate a wide range of cellular functions including cell survival, and the proliferation ${ }^{37)}$ and inhibition of the PI3K/AKT pathway have noticeable cytotoxic effects. ${ }^{41)}$

The activation of MAPKs p38 and JNK1/2 also had an inhibitory effect on the proliferation and growth of prostate, colon, and osteosarcoma cancer cell lines. ${ }^{36)}$ After treatment with the synthetic SL analogues (MEB55 and ST362), there was a marked decrease in the stability of cyclin B1 protein, which was found to be due to the reduced synthesis of cyclin B1 mRNA and pro- tein degradation, thus causing cancer cells to be arrested at the $\mathrm{G} 2 / \mathrm{M}$ phase of the cell cycle. To further examine the role of p38 and JNK1/2 MAPKs in growth inhibition and apoptosis, cancer cell lines were administered with inhibitors of p38 and JNK1/2 preceding treatment with MEB55. It was observed that the inhibition by this SL analogue was significantly reduced. Cyclin G2, which is found to be induced by therapeutics involving DNA damage and is associated with growth inhibition, was improved by MEB55 and ST362. The expression of BIRC3, which is an inhibitor of apoptosis, was found to be decreased, while that of DDIT3, a transcription factor that is activated by stress (which includes DNA damage) was found to be increased in cells treated with the same synthetic SL analogues. ${ }^{36}$ )

Synthetic SL analogues also exhibited inhibition of a xenograft model of breast cancer. The treatment of mice implanted with breast cancer xenografts with MEB55 showed reduced growth rates and volume of the tumors. Interestingly, when combined with paclitaxel, it showed an additive effect on the growth inhibition of breast cancer cell lines. Similar to paclitaxel, these synthetic SL analogues were found to hinder cell motility of breast cancer cells by affecting the integrity of microtubules through microtubule bundling, hence inhibiting the proliferation and migration of breast cancer cells, ${ }^{3)}$ thus proving to be anti-cancer agents.

It was recently reported that ST362 and MEB55 destabilize genomic DNA, induce DNA double-strand breaks (DSBs) and activate DNA damage response in osteosarcoma cells but simultaneously inhibit DNA repair, resulting in cell death. These

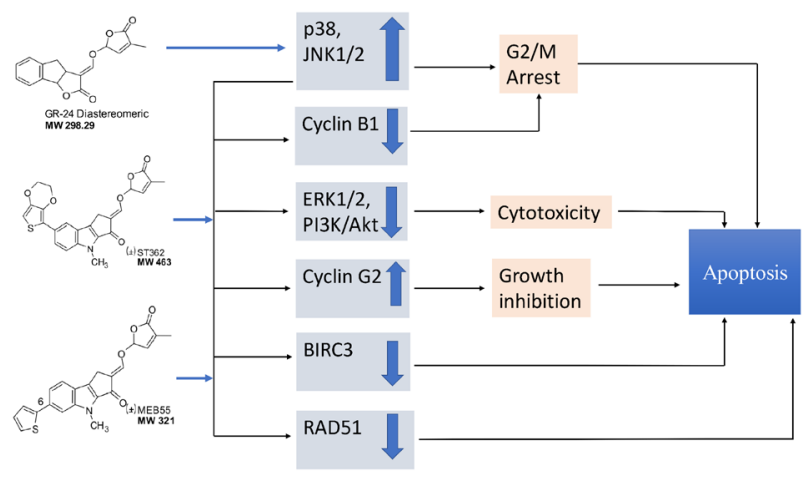

Fig. 2. Schematic view depicting the differential regulation of genes and biological processes related to cell death and apoptosis induced by GR-24, ST362 and MEB55. 


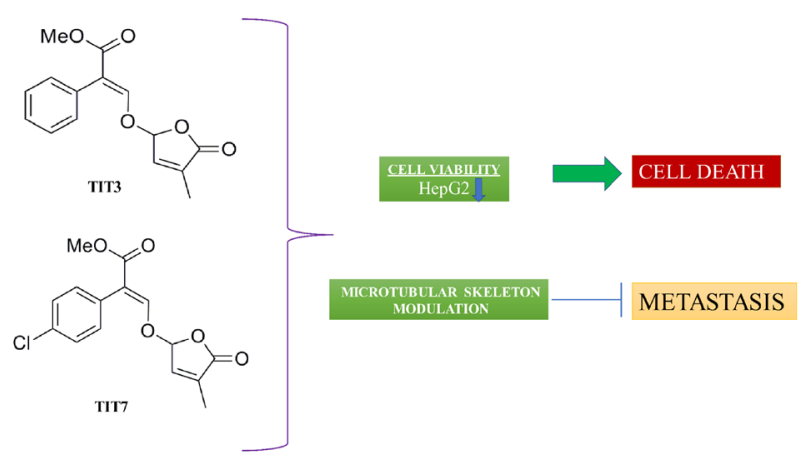

Fig. 3. Overview of the anti-cancer effects exerted by the novel strigolactone analogues TIT 3 and TIT 7 in HepG2 cells.

findings, quite interestingly, reported that these synthetic SL analogues failed to either cause any DSBs or cells deaths in nontransformed BJ fibroblast cells, suggesting their essential future clinical relevance. These synthetic SL analogues were found to downregulate the expression of RAD51 via ubiquitination in a proteasome-dependent manner, thereby inhibiting RAD51 relocalization to DSB sites. ${ }^{42)}$ RAD51 is a repair protein that colocalizes at DSB sites following DNA damage, the overexpression of which corresponds to an increase in DNA repair activity that could result in resistance to DNA-damaging chemotherapy and radiotherapy. ${ }^{43)}$ The inhibition of RAD51 in cancer cells causes the downregulation of homology-directed repair (HDR), a prominent DNA repair pathway, hence leading to the death of cancer cells. Therefore, the downregulation of RAD51 could enhance the effectiveness of cancer therapies. Intriguingly, ST362 and MEB55 further sensitize cells to chemotherapeutic drugs such as PARP inhibitors to improve their efficacy once RAD51 (hence HDR) is inhibited. ${ }^{42,43)}$ Figure 2 represents a schematic diagram of the mechanism of the action of GR-24, ST362 and MEB55 on cancer cells leading to apoptosis.

In our recent study, we assessed the anti-proliferative and proapoptotic effect of two newly synthesized SL analogues, TIT3 and TIT7, on HepG2 (hepatocellular carcinoma) and Jurkat (acute lymphoblastic leukemia) cell lines. ${ }^{44)}$ It was interesting to note that these two analogues, significantly reduced the viability of cancer cells with only a miniscule inhibition on normal BHK (baby hamster kidney) cells. Moreover, both TIT3 and TIT7 inhibited the migration of HepG2 cells (Fig. 3) in a wound healing/scratch assay, which suggests that TIT3 and TIT7 also affect the organization of microtubules. ${ }^{44)}$ However, we need further investigations to decipher the exact molecular mechanisms involved.

\section{Conclusion}

The above findings suggest that synthetic SL analogues could be promising anti-cancer drug candidates for designing a cancer therapy that induces apoptosis via cellular stress, cell cycle arrest, the destabilization of microtubules and the downregulation of HDR.

Moreover, as there are a variety of plants synthesizing differ- ent types of SLs in varying quantities, it is possible to further synthesize different analogues for the treatment of a broader range of cancer types. Synthetic SL analogues have not been found to have any of the cytotoxic effects against healthy cells usually seen with chemotherapy drugs. Moreover, besides being anti-cancerous, these synthetic SL analogues have been shown to enhance the efficiency of chemotherapy drugs by an additive effect. The action mechanism of these analogues against a broader range of cancer cells still remains obscure, and the current findings could form a link in the discovery and integration of future discoveries to make these analogues more effective.

\section{Conflict of interest}

The authors declare that they have no financial conflict of interest.

\section{References}

1) J. Ferlay, I. Soerjomataram, R. Dikshit, S. Eser, C. Mathers, M. Rebelo, D. M. Parkin, D. Forman and F. Bray: Int. J. Cancer 136, E359E386 (2015).

2) M. Dobbelstein and U. Moll: Nat. Rev. Drug Discov. 13, 179-196 (2014).

3) E. Mayzlish-Gati, D. Laufer, C. F. Grivas, J. Shaknof, A. Sananes, A. Bier, S. Ben-Harosh, E. Belausov, M. D. Johnson, E. Artuso, O. Levi, O. Genin, C. Prandi, I. Khalaila, M. Pines, R. I. Yarden, Y. Kapulnik and H. Koltai: Cancer Biol. Ther. 16, 1682-1688 (2015).

4) H. Easwaran, H.-C. Tsai and S. B. Baylin: Mol. Cell 54, 716-727 (2014).

5) A. Singh and J. Settleman: Oncogene 29, 4741-4751 (2010).

6) L. Menger, E. Vacchelli, O. Kepp, A. Eggermont, E. Tartour, L. Zitvogel, G. Kroemer and L. Galluzzi: OncoImmunology 2, e23082 (2013).

7) A. D. Kinghorn: J. Pharm. Pharmacol. 53, 135-148 (2001).

8) M. J. Balunas and A. D. Kinghorn: Life Sci. 78, 431-441 (2005).

9) Y.-W. Chin, M. J. Balunas, H. B. Chai and A. D. Kinghorn: AAPS J. 8, E239-E253 (2006).

10) R. J. Krueger: J. Nat. Prod. 68, 631 (2005).

11) D. J. Newman and G. M. Cragg: Curr. Med. Chem. 11, 1693-1713 (2004).

12) A. B. Kunnumakkara, P. Anand and B. B. Aggarwal: Cancer Lett. 269, 199-225 (2008).

13) Y. E. Marin, B. A. Wall, S. Wang, J. Namkoong, J. J. Martino, J. Suh, H. J. Lee, A. B. Rabson, C. S. Yang, S. Chen and J. H. Ryu: Melanoma Res. 17, 274-283 (2007).

14) R. L. Thangapazham, S. Sharad and R. K. Maheshwari: Biofactors 39, 141-149 (2013).

15) S. J. Chatterjee and S. Pandey: Cancer Biol. Ther. 11, 216-228 (2011).

16) J. Cao, Y. Liu, L. Jia, H. M. Zhou, Y. Kong, G. Yang, L. P. Jiang, Q. J. Li and L. F. Zhong: Free Radic. Biol. Med. 43, 968-975 (2007).

17) K. K. Gupta, S. S. Bharne, K. Rathinasamy, N. R. Naik and D. Panda: FEBS J. 273, 5320-5332 (2006).

18) M. C. Wani, H. L. Taylor, M. E. Wall, P. Coggon and A. T. McPhail: J. Am. Chem. Soc. 93, 2325-2327 (1971).

19) W. N. Hait, E. Rubin, E. Alli and S. Goodin: Update Cancer Ther. 2, 1-18 (2007).

20) A. E. Prota, K. Bargsten, D. Zurwerra, J. J. Field, J. F. Díaz, K. H. Altmann and M. O. Steinmetz: Science 339, 587-590 (2013).

21) K. Priyadarshini and U. Keerthi Aparajitha: Med. Chem. 2, 139-141 (2012).

22) E. P. Bruggemann, S. J. Currier, M. M. Gottesman and I. Pastan: J. 
Biol. Chem. 267, 21020-21026 (1992).

23) V. K. Ngan, K. Bellman, D. Panda, B. T. Hill, M. A. Jordan and L. Wilson: Cancer Res. 60, 5045-5051 (2000).

24) F. Skoog, F. M. Strong and C. O. Miller: Science 148, 532-533 (1965).

25) Y. Ishii, S. Sakai and Y. Honma: Biochim. Biophys. Acta 1643, 11-24 (2003).

26) J. Voller, M. Zatloukal, R. Lenobel, K. Dolezal, T. Béres, V. Krystof, L. Spíchal, P. Niemann, P. Dzubák, M. Hajdúch and M. Strnad: Phytochemistry 71, 1350-1359 (2010).

27) J. Steigerova, J. Okleštková, M. Levková, L. Rárová, Z. Kolář and M. Strnad: Chem. Biol. Interact. 188, 487-496 (2010).

28) S. Cohen and E. Flescher: Phytochemistry 70, 1600-1609 (2009).

29) V. Gomez-Roldan, S. Fermas, P. B. Brewer, V. Puech-Pagès, E. A. Dun, J. P. Pillot, F. Letisse, R. Matusova, S. Danoun, J. C. Portais, H. Bouwmeester, G. Bécard, C. A. Beveridge, C. Rameau and S. F. Rochange: Nature 455, 189-194 (2008).

30) M. Umehara, A. Hanada, S. Yoshida, K. Akiyama, T. Arite, N. Takeda-Kamiya, H. Magome, Y. Kamiya, K. Shirasu, K. Yoneyama, J. Kyozuka and S. Yamaguchi: Nature 455, 195-200 (2008).

31) C. E. Cook, L. P. Whichard, B. Turner, M. E. Wall and G. H. Egley: Science (80-.). 154, 1189-1190 (1966).

32) X. Xie, K. Yoneyama and K. Yoneyama: Annu. Rev. Phytopathol. 48, 93-117 (2010).

33) K. Akiyama, S. Ogasawara, S. Ito and H. Hayashi: Plant Cell Physiol. 51, 1104-1117 (2010).

34) Y. Kapulnik, P. M. Delaux, N. Resnick, E. Mayzlish-Gati, S. Wininger,
C. Bhattacharya, N. Séjalon-Delmas, J. P. Combier, G. Bécard, E. Belausov, T. Beeckman, E. Dor, J. Hershenhorn and H. Koltai: Planta 233, 209-216 (2011).

35) C. Rameau: C. R. Biol. 333, 344-349 (2010).

36) C. B. Pollock, S. McDonough, V. S. Wang, H. Lee, L. Ringer, X. Li, C. Prandi, R. J. Lee, A. S. Feldman, H. Koltai, Y. Kapulnik, O. C. Rodriguez, R. Schlegel, C. Albanese and R. I. Yarden: Oncotarget 5, 16831698 (2014).

37) C. B. Pollock, H. Koltai, Y. Kapulnik, C. Prandi and R. I. Yarden: Breast Cancer Res. Treat. 134, 1041-1055 (2012).

38) C. Correze, J.-P. Blondeau and M. Pomerance: Eur. J. Endocrinol. 153, 123-133 (2005).

39) K. Iyoda, Y. Sasaki, M. Horimoto, T. Toyama, T. Yakushijin, M. Sakakibara, T. Takehara, J. Fujimoto, M. Hori, J. R. Wands and N. Hayashi: Cancer 97, 3017-3026 (2003).

40) H.-L. Chang, Y.-C. Wu, J.-H. Su, Y.-T. Yeh and S.-S. F. Yuan: J. Pharmacol. Exp. Ther. 325, 841-849 (2008).

41) U. Elia and E. Flescher: Neoplasia 10, 1303-1313 (2008).

42) M. P. Croglio, J. M. Haake, C. P. Ryan, V. S. Wang, J. Lapier, J. P. Schlarbaum, Y. Dayani, E. Artuso, C. Prandi, H. Koltai et al.: Oncotarget 7, 13984-14001 (2016).

43) A. Ward, K. K. Khanna and A. P. Wiegmans: Cancer Treat. Rev. 41, 35-45 (2015).

44) M. N. Hasan, H. Choudhry, S. S. Razvi, S.S. Moselhy, T. A. Kumosani et al.: Bioorg. Med. Chem. Lett. 28, 1077-1083 (2018). 